\title{
mRNA COVID-19 Vaccines and Potential Autoimmunity
}

\author{
Ali Amanati ${ }^{1}$ and Mazyar Ziyaeyan ${ }^{1, *}$ \\ ${ }^{1}$ Professor Alborzi Clinical Microbiology Research Center, Shiraz University of Medical Sciences, Shiraz, Iran \\ "Corresponding author: Professor Alborzi Clinical Microbiology Research Center, Shiraz University of Medical Sciences, Shiraz, Iran. Email: ziyaeyan@gmail.com
}

Received 2021 June 26; Accepted 2021 July 10.

Keywords: Autoimmunity, mRNA COVID-19 Vaccine, SARS-CoV-2

\section{Dear editor,}

Recently, Zhang et al. (1) published an article on the reverse-transcribed severe acute respiratory syndrome coronavirus 2 (SARS-CoV-2) RNA integration into the genome of cultured human cells. In this exciting article, the authors concluded that transcription of the integrated sequences might account for some of the positive polymerase chain reaction (PCR) tests seen in patients with prolonged detection of SARS-CoV-2 RNA and repeated PCR-positive tests after recovery from COVID-19. The authors demonstrated that the SARS-CoV-2 nucleocapsid (NC) sequences can be detected and cloned after reversetranscription of the virus RNA and integration of the resulting DNA into the host cell's genome.

In this regard, we believe that several important aspects need to be considered and further investigated. First of all, should we consider any long-term impact by the potential genomic reverse-transcription of the virus RNA and integration in the infected host? Second, how long may this phenomenon last? Third, could this phenomenon happen universally in all infected patients with different disease stages? Fourth, is it comparable in different host tissues? Indeed, more reliable data is needed to address these unquestioned issues.

Besides, the authors also raise additional paramount concern regarding the potential triggering of the autoimmunity by viral neoantigen after recovery of the infection.

The viral-induced autoimmune disease is a well-known phenomenon previously described by many researchers after common viral infections (2). It is also currently reintroduced by the SARS-CoV-2 infection $(3,4)$. This potential possibility after the virus genomic integration could garner attention because of its implications regarding similar hypothetical consequences after massive immunization by the available new mRNA COVID-19 vaccines.
It is premature to draw any conclusions about the likelihood of the post-mRNA COVID-vaccine induced autoimmunity based on this report; however, the threshold for the production of viral neoantigen by the genome that enters the cell by mRNA vaccines should be further investigated. Another question is that if it could happen, how long can this genome pave the way for neo-proteins production in the host after vaccination?

If this protein production exceeds its threshold, it can affect the host's immune system for an unknown period and more than expected, leading to disease and side effects or weakening of the host.

\section{Footnotes}

Authors' Contribution: Study concept and design: AA. Drafting the manuscript: AA and MZ. Critical revision of the manuscript for valuable intellectual content: AA and MZ.
Conflict of Interests: None
Ethical Approval: Not applicable
Funding/Support: None

\section{References}

1. Zhang L, Richards A, Barrasa M, Hughes SH, Young RA, Jaenisch R. Reverse-transcribed SARS-CoV-2 RNA can integrate into the genome of cultured human cells and can be expressed in patient-derived tissues. Proceed National Acad Sci. 2021;118(21). doi: 10.1073/pnas.2105968118.

2. Smatti MK, Cyprian FS, Nasrallah GK, Al Thani AA, Almishal RO, Yassine HM. Viruses and Autoimmunity: A Review on the Potential Interaction and Molecular Mechanisms. Viruses. 2019;11(8). doi: 10.3390/v11080762. [PubMed: 31430946]. [PubMed Central: PMC6723519].

3. Bhattacharjee S, Banerjee M. Immune Thrombocytopenia Secondary to COVID-19: a Systematic Review. SN Compr Clin Med. 2020:1-11. doi: 
10.1007/s42399-020-00521-8. [PubMed: 32984764]. [PubMed Central: PMC7501509].

4. Canas CA. The triggering of post-COVID-19 autoimmunity phenomena could be associated with both transient immunosup- pression and an inappropriate form of immune reconstitution in susceptible individuals. Med Hypotheses. 2020;145:110345. doi: 10.1016/j.mehy.2020.110345. [PubMed: 33080459]. [PubMed Central: PMC7556280]. 